SLAC-PUB-8281

December 1999

\title{
The Three Loop Slope of the Dirac Form Factor and the $S$ Lamb Shift in Hydrogen
}

\author{
Kirill Melnikov \\ Stanford Linear Accelerator Center \\ Stanford University, Stanford, CA 94309 \\ Timo van Ritbergen \\ Institut fur Theoretische Teilchenphysik, \\ Universitat Karlsruhe, D-76128 Karlsruhe, Germany
}


SLAC-PUB-8281, TTP99-44

thep-ph $2991127 \%$

\title{
The three loop slope of the Dirac form factor and the $1 S$ Lamb shift in hydrogen
}

\author{
Kirill Melnikov* \\ Stanford Linear Accelerator Center \\ Stanford University, Stanford, CA 94309 \\ Timo van Ritbergen ${ }^{\dagger}$ \\ Institut für Theoretische Teilchenphysik, \\ Universität Karlsruhe, D-76128 Karlsruhe, Germany
}

\begin{abstract}
The last unknown contribution to hydrogen energy levels at order $m \alpha^{7}$, due to the slope of the Dirac form factor at three loops, is evaluated in a closed analytical form. The resulting shift of the hydrogen $n \mathrm{~S}$ energy level is found to be $3.016 / n^{3} \mathrm{kHz}$. Using the QED calculations of the $1 S$ Lamb shift, we extract a precise value of the proton charge radius $r_{p}=0.883 \pm 0.014 \mathrm{fm}$.
\end{abstract}

Precision experiments with hydrogen and, more generally, with hydrogen-like atoms serve as an excellent laboratory to test theoretical approaches to bound state QED (for a recent review see, e.g. [1]1] $]_{1}^{-1}$ ). These experiments address a number of features of the simplest atoms, such as the energy levels of the ground and excited states, and the corresponding lifetimes.

In recent years we have seen remarkable progress in the experimental study of the hydrogen atom. In particular the accuracy of the $1 \mathrm{~S}$ Lamb shift measurements

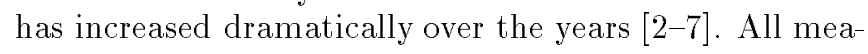
surements are consistent with each other and the most accurate value so far was determined in Ref. [1] [1]:

$$
\Delta E(1 S)_{\exp }=8172837(22) \mathrm{kHz}
$$

This result was obtained by analysing the most precise measurements for the transition frequencies in hydrogen (see [in for details).

Theoretically, since the ratio of the electron mass $m$ to the proton mass $M$ is very small, it is convenient to write hydrogen energy levels as a double expansion in $\alpha$ and $m / M$. The corrections which survive in the limit $M \rightarrow \infty$ are known as non-recoil corrections, the other corrections are called recoil ones. It is further convenient to organize non-recoil corrections in powers of $\alpha^{n}(Z \alpha)^{l}$, assigning an auxiliary notation $Z$ for the proton charge. In this case the correction $\alpha^{n}(Z \alpha)^{l}$ describes a contribution of all diagrams with $l-3$ Coulomb photons exchanged between the electron and the proton and with

\footnotetext{
*e-mail: melnikov@slac.stanford.edu

${ }^{\dagger}$ e-mail: timo@particle.physik.uni-karlsruhe.de
}

$n$ photons emitted and absorbed by the electron. The general expression for the $n \mathrm{~S}$-level shift can be written as:

$$
\Delta E=\Delta E_{\text {non-recoil }}+\Delta E_{\text {recoil }}+\Delta E_{\mathrm{vp}}+\Delta E_{\text {proton }},
$$

where we have also added a contribution of muons and hadrons to photon vacuum polarization and a contribution due to the proton structure (see a discussion below).

We begin with non-recoil corrections. These corrections can be parameterized as (see e.g. [ [ $\left[\begin{array}{c}1 \\ 1\end{array}\right]\left[\begin{array}{l}1 \\ 1\end{array}\right]$ ):

$$
\begin{aligned}
\Delta E_{\text {non-recoil }} & =\frac{m \alpha(Z \alpha)^{4}}{\pi n^{3}}\left(\frac{m_{r}}{m}\right)^{3}\left(A_{40}+A_{41} L+(Z \alpha) A_{50}\right. \\
& \left.+(Z \alpha)^{2}\left[A_{62} L^{2}+A_{61} L+A(Z \alpha)\right]\right) \\
& +\frac{m \alpha^{2}(Z \alpha)^{4}}{\pi^{2} n^{3}}\left(\frac{m_{r}}{m}\right)^{3}\left(B_{40}+(Z \alpha) B_{50}\right) \\
& +\frac{m \alpha^{3}(Z \alpha)^{4}}{\pi^{3} n^{3}}\left(\frac{m_{r}}{m}\right)^{3} C_{40}+\mathcal{O}\left(m \alpha^{8} \log ^{3} \alpha\right)
\end{aligned}
$$

where $m_{r}=m M /(m+M)$ is the reduced mass of the electron and $L=\log m /\left(m_{r}(Z \alpha)^{2}\right)$ and the function $A(Z \alpha)$ contains all higher order terms in the expansion in $Z \alpha$. We have also indicated that the higher order corrections contain a logarithm of the fine structure constant in the third power [i1 1].

All the terms in the above equation, with the exception for $C_{40}$, are currently known. It is the purpose of this paper to report on the calculation of the last missing ingredient in $C_{40}$, the slope of the Dirac form factor at zero momentum transfer.

The hydrogen atom is formed because of a Coulomb interaction of the proton with the electron. The interaction of the virtual photon with the electron on its mass shell can be parameterized by the so-called Dirac and Pauli form factors:

$$
\bar{u}\left(p_{2}\right) \Gamma_{\mu} u\left(p_{1}\right)=\bar{u}\left(p_{2}\right)\left(F_{1}\left(q^{2}\right) \gamma_{\mu}+i \sigma_{\mu \nu} \frac{q_{\mu}}{m} F_{2}\left(q^{2}\right)\right) u\left(p_{1}\right),
$$

where the $u(p)$ are the electron spinors in the initial and final state and $q$ is the momenta carried away by the photon. The momenta satisfy the relation $q=p_{2}-p_{1}$. An important consequence of QED gauge invariance and the electron charge definition is that the Dirac form factor equals unity at zero momentum transfer $F_{1}(0)=1$, to all orders in the coupling constant. The Pauli form factor at 
zero momentum transfer describes an interaction of the electron spin with the homogeneous magnetic field; it is the electron anomalous magnetic moment.

Let us now turn to the contribution of the Dirac form factor to hydrogen energy levels. The typical momenta of the Coulomb photon in hydrogen is given by the inverse Bohr radius $q_{\text {typ }} \sim m \alpha$, where $m$ is the electron mass. Compared to the electron mass, this momentum transfer is quite small and for this reason one can Taylor expand the Dirac form factor in powers of $q^{2} / \mathrm{m}^{2}$. One obtains:

$$
F_{1}\left(q^{2}\right)=1+F_{1}^{\prime} \frac{q^{2}}{m^{2}}+\mathcal{O}\left(\frac{q^{4}}{m^{4}}\right)
$$

where, as we already mentioned, the first term equals unity to all orders in the coupling constant. The slope of the Dirac form factor, $F_{1}^{\prime}$ in the above formula, can be written as a series in $\alpha$ :

$$
F_{1}^{\prime}=\sum_{n=1}^{\infty}\left(\frac{\alpha}{\pi}\right)^{n} A_{\text {slope }}^{(n)}
$$

In this paper we consider the first three terms in this series.

Two things should be noted at this point. The first and second order corrections to the slope of the Dirac form factor contribute to the coefficients $A_{40}$ and $B_{40}$ in

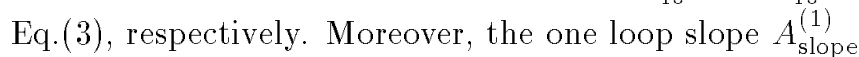
is not infrared finite, as can be seen from the fact that the term $A_{41} \log (\alpha)$ appears in Eq. $\left(\begin{array}{c}\left(\hat{\beta}_{1}^{1}\right. \\ 1\end{array}\right)$. This infrared divergence gets removed if one takes into account that the electron is not on its mass shell in the bound state. In the language of the effective theories, the one loop slope in Eq. ( $(4-1)$ corresponds to "hard" contributions to energy levels. Since the two and three loop slopes of the Dirac form factor are infrared finite, the off-shellness of the electron is irrelevant for these contributions.

Being divergent, the one loop slope depends upon the chosen regularization. In the present calculation we have used dimensional regularization (the space-time dimension is $D=4-2 \epsilon$ ) for both ultraviolet and infrared divergences.

Let us briefly describe how the actual calculation of $A_{\text {slope }}^{(3)}$ has been done. After applying a projection operator for the Dirac form factor on the electron-photon vertex and after performing the Taylor expansion in the photon momentum transfer, one obtains diagrams of the self energy type. There are four basic topologies which appear in this calculation, characteristic example diagrams are depicted in Fig.1. For each of the topologies one writes down a system of recurrence relations obtained by the use of integration-by-parts [12]. Solving this system, it is possible to show that any integral which belongs to the above topologies can be expressed through 17 master integrals. Luckily, these seventeen integrals have already been computed in the course of the analytical calculation of the electron anomalous magnetic moment [1] $\left.\overline{3}_{1}\right]$ and hence can be taken from there. Let us mention, that as a check of the calculation, and in particular on our solution of the system of recurrence relations, we have reproduced also the analytical value of the three-loop electron anomalous magnetic moment. Details of our calculation, including the results for the individual diagrams, will be presented in a separate publication.

Our result for the slope of the Dirac form factor is

$$
\begin{aligned}
A_{\text {slope }}^{(1)}= & -\frac{1}{8}-\frac{1}{6 \epsilon} \\
A_{\text {slope }}^{(2)}= & -\frac{4819}{5184}-\frac{3}{4} \zeta_{3}+\frac{1}{2} \pi^{2} \log 2-\frac{49}{432} \pi^{2} \\
A_{\text {slope }}^{(3)}= & -\frac{77513}{186624}-\frac{17}{24} \zeta_{3} \pi^{2}-\frac{2929}{288} \zeta_{3}+\frac{25}{8} \zeta_{5} \\
& +\frac{41671}{2160} \pi^{2} \log 2-\frac{103}{1080} \pi^{2} \log ^{2} 2-\frac{454979}{38880} \pi^{2} \\
& +\frac{3899}{25920} \pi^{4}-\frac{217}{216} \log ^{4} 2-\frac{217}{9} a_{4}
\end{aligned}
$$

where $a_{4}=\sum_{n=1}^{\infty} 1 /\left(2^{n} n^{4}\right)$ and $\zeta_{k}=\sum_{n=1}^{\infty} 1 /\left(n^{k}\right)$ denotes the Riemann zeta function.

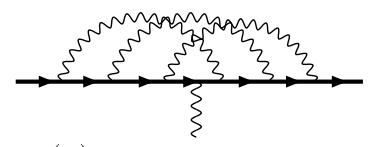

(a)

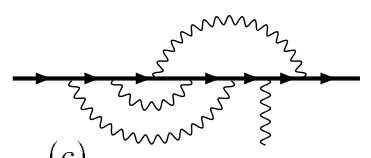

(c)

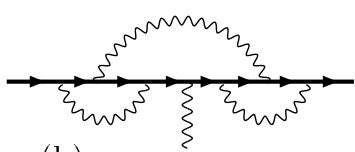

(b)

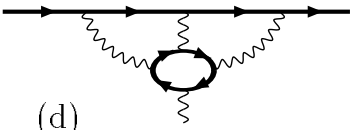

FIG. 1. Examples of electron-photon vertex diagrams that correspond to the four different integration topologies.

We now turn to the contribution of the slope of the Dirac form factor to hydrogen energy levels. It is easy to see that the slope of the Dirac form factor gives rise to a perturbation of the Coulomb potential:

$$
\delta V_{\text {slope }}(r)=\frac{4 \pi Z \alpha}{m^{2}} \delta^{3}(\boldsymbol{r}) F_{1}^{\prime} .
$$

This perturbation delivers a $n$ S-level energy shift:

$$
\Delta E_{\text {slope }}=\left\langle\psi(r)\left|\delta V_{\text {slope }}(r)\right| \psi(r)\right\rangle=\frac{4 \pi \alpha Z}{m^{2}} F_{1}^{\prime}|\psi(0)|^{2} \text {. }
$$

Here $|\psi(0)|^{2}=\left(m_{r} \alpha Z\right)^{3} /\left(\pi n^{3}\right)$ is the square of the hydrogen wave function at the origin. The correction induced by the three-loop slope of the Dirac form factor is then $n_{-}^{12}$.

\footnotetext{
${ }^{1}$ The first correct numerical result on the two loop slope of the Dirac form factor was published in Ref. [1, 1, The analytical result was obtained in [1, 1 i 1 .

${ }^{2}$ In what follows numerical results are given in frequency units using $\Delta E \rightarrow \Delta E /(2 \pi \hbar)$.
} 


$$
\begin{aligned}
\Delta E_{\alpha^{3}(Z \alpha)^{4}}^{\text {slope }}(n) & =\frac{4 m \alpha^{7}}{\pi^{3} n^{3}}\left(\frac{m_{r}}{m}\right)^{3} A_{\text {slope }}^{(3)} \\
& \rightarrow \frac{8 c R_{\infty} \alpha^{5}}{\pi^{3} n^{3}}\left(\frac{m_{r}}{m}\right)^{3} A_{\text {slope }}^{(3)}
\end{aligned}
$$

where $c R_{\infty}$ is the Rydberg constant in $\mathrm{MHz}$.

Using the values for the Rydberg and the fine structure constant $[1] 6$

$$
\begin{aligned}
& c R_{\infty}=3289841960.367(25) \mathrm{MHz}, \\
& \alpha=1 / 137.03599976(50),
\end{aligned}
$$

we arrive at:

$$
\Delta E_{\alpha^{3}(Z \alpha)^{4}}^{\text {slope }}(n)=\frac{3.016}{n^{3}} \mathrm{kHz} .
$$

The contribution due to the slope of the Dirac form factor was the last unknown contribution to the hydrogen energy levels at order $\alpha^{3}(Z \alpha)^{4}$. The two other contributions to the coefficient $C_{40}$ come from the three-loop electron anomalous magnetic moment and the three-loop vacuum polarization correction to the Coulomb propagator. These contributions can be extracted from the

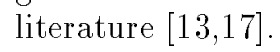

Taking all three contributions into account, we obtain the following expression for the coefficient $C_{40}$ for the S-levels:

$$
\begin{aligned}
C_{40} & =\frac{679441}{93312}-\frac{252251}{9720} \pi^{2}+\frac{4787}{108} \pi^{2} \log 2 \\
& -\frac{121}{72} \zeta_{3} \pi^{2}-\frac{239}{135} \pi^{2} \log ^{2} 2-\frac{84071}{2304} \zeta_{3}+\frac{85}{24} \zeta_{5} \\
& -\frac{568}{9} a_{4}+\frac{1591}{3240} \pi^{4}-\frac{71}{27} \log ^{4} 2 \approx 0.417508
\end{aligned}
$$

which results in a three-loop correction to the $n \mathrm{~S}$-level Lamb shift:

$$
\begin{aligned}
& \Delta E_{\alpha^{3}(Z \alpha)^{4}}(n)=\frac{m \alpha^{7}}{n^{3} \pi^{3}}\left(\frac{m_{r}}{m}\right)^{3} C_{40} \\
& =(3.016+5.187-6.370) \frac{\mathrm{kHz}}{n^{3}}=\frac{1.83}{n^{3}} \mathrm{kHz} .
\end{aligned}
$$

In the above equation we have displayed the contributions due to the three-loop slope of the Dirac form factor, the three-loop anomalous magnetic moment of the electron and the three-loop photon vacuum polarization function separately to emphasize a strong cancellation which occurs between vertex and vacuum polarization corrections. Thanks to this cancellation, the correction turns out to be quite small numerically.

We now turn to other results available in the literature.

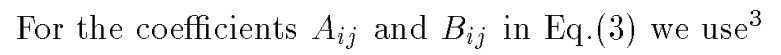

\footnotetext{
${ }^{3}$ See Ref. [8] for reference to earlier work. Some of the numbers in Ref. [R have been updated according to recent calculations in Refs. [1 $18+20]$. The value of the non-perturbative function $A(1 S)(Z \alpha)$ for $Z=1$ is extracted from Ref. [12 1. for the self energy correction and from Ref. [990 for the vacuum polarization.
}

$$
\begin{aligned}
& A_{40}(n S)=\frac{10}{9}-\frac{4}{15}-\frac{4}{3} \log k_{0}(n S), \quad A_{41}(n S)=\frac{4}{3}, \\
& \log k_{0}(1 S)=2.9841285557655(1), \\
& A_{50}(n S)=\pi\left(\frac{139}{32}+\frac{5}{48}-2 \log 2\right), \quad A_{62}(n S)=-1, \\
& A_{61}(1 S)=\frac{28}{3} \log 2-\frac{21}{20}-\frac{2}{15}, \\
& A(1 S)(Z \alpha)=-30.29024(2)--0.6187+\left(\frac{19}{45}-\frac{\pi^{2}}{27}\right), \\
& B_{40}(n S)=-\frac{2179}{648}-\frac{9}{4} \zeta_{3}+\frac{3}{2} \pi^{2} \log 2-\frac{10}{27} \pi^{2}, \\
& B_{50}(1 S)=-21.558(3) .
\end{aligned}
$$

The only known non-recoil correction at order $\mathcal{O}\left(m \alpha^{8}\right)$ is the triple logarithmic enhanced contribution [1]

$$
\Delta E_{\alpha^{2}(Z \alpha)^{6}}=-\frac{8}{27} \frac{m \alpha^{2}(Z \alpha)^{6}}{\pi^{2} n^{3}} \log ^{3} \frac{1}{(Z \alpha)^{2}}=-\frac{28.4}{n^{3}} \mathrm{kHz} .
$$

This correction is included in the central value for the $1 \mathrm{~S}$ Lamb shift quoted below.

Consider now the recoil corrections. Part of these are already included in Eq. (13ij) through its dependence on the reduced mass of the electron. The terms which go beyond this approximation are:

$$
\begin{aligned}
\Delta E_{\text {recoil }} & =\frac{m^{2}(Z \alpha)^{5}}{\pi M}\left(\frac{1}{3} L-\frac{8}{3} \log k_{0}(1 S)+\frac{62}{9}+\frac{14}{3} \log 2\right. \\
& \left.-\frac{2}{M^{2}-m^{2}}\left(M^{2} \log \frac{m}{m_{r}}-m^{2} \log \frac{M}{m_{r}}\right)\right) \\
+ & \frac{m^{2}(Z \alpha)^{6}}{M}\left(4 \log 2-\frac{7}{2}\right)-\frac{\alpha(Z \alpha)^{5} m^{2}}{\pi^{2} M}(1.36449(2)) .
\end{aligned}
$$

computed in Refs. [22 24$]$.

The vacuum polarization effects due to muons and hadrons can be extracted from Ref. [225] and give $\Delta E_{\mathrm{vp}}=$ $-8.5 \mathrm{kHz}$ for the $1 \mathrm{~S}$ Lamb shift.

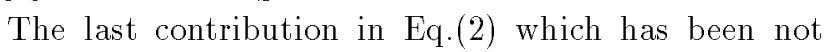
considered so far is the one due to proton structure. The major part of this correction to the Lamb shift is parameterized through the proton charge radius but there is also a proton self energy correction which goes beyond that approximation [24]. The complete correction reads:

$$
\begin{aligned}
\Delta E_{\text {proton }}= & \frac{2(Z \alpha)^{4}}{3 n^{3}}\left\langle r_{p}^{2}\right\rangle m_{r}^{3}+\frac{4 m_{r}^{3}}{3 \pi n^{3} M^{2}}\left(Z^{2} \alpha\right)(Z \alpha)^{4} \\
& \times\left[\log \frac{M}{m_{r}(Z \alpha)^{2}}-\log k_{0}(n)\right] .
\end{aligned}
$$

There are two different values for the proton charge radius $r_{p}=0.805(11) \mathrm{fm}$ and $r_{p}=0.862(12)$ fm obtained in $\left[2 \overline{6}_{6}, 2 \overline{2}\right]$, respectively, by analysing the electron proton scattering data. The smaller value, $r_{p}=0.805(11) \mathrm{fm}$, seems to give a serious disagreement between precision atomic measurements and the theoretical predictions [2] $\bar{y}_{1}^{\prime}$. For this reason we do not consider it here. The 
data of Ref. [2] $\left.\tilde{2}_{1}\right]$, on the other hand, was recently reanalized in [28] , where a normalization of the proton charge form factor at zero momenta transfer was also treated as a free parameter in the fit. The analysis of Ref. [228] leads to a larger proton charge radius $r_{p}=0.877(24) \mathrm{fm}$. The final value for the $1 \mathrm{~S}$-shift strongly depends on the value of the proton charge radius:

$$
\begin{aligned}
& \Delta E(1 S)_{\text {theory }}=8172778(16)(32) \mathrm{kHz}, \\
& \Delta E(1 S)_{\text {theory }}=8172819(16)(66) \mathrm{kHz},
\end{aligned}
$$

where the values are given for $r_{p}=0.862(12) \mathrm{fm}$ and $r_{p}=0.877(24) \mathrm{fm}$, respectively. The first error in all the above equations is the theoretical uncertainty due to still uncalculated higher order corrections to energy levels beyond the $\alpha^{7}$ order (see a discussion below). The second error is due to the uncertainty in the experimental values of the proton charge radius. The uncertainties in Rydberg and the fine structure constants are not relevant at the present level of precision.

Comparing these numbers with the most recent measurement of the 1S-level Lamb shift [i]]

$$
\Delta E(1 S)_{\text {exp }}=8172837(22) \mathrm{kHz} \text {. }
$$

we conclude that the larger values for the proton charge radius seem to give an agreement between the theory and experiment.

An important part of the error in $\Delta E(1 S)_{\text {theory }}$ is due to uncalculated higher order corrections. For the $1 S$ Lamb shift the uncertainty $\left[2{ }^{2} 9^{4}\right]$ was estimated to be about $40 \mathrm{kHz}$. This number is the linear sum of a $8 \mathrm{kHz}$ uncertainty in the self energy correction, the $16 \mathrm{kHz}$ uncertainty caused by the unknown $\alpha^{2}(Z \alpha)^{6}$ terms and the $16 \mathrm{kHz}$ uncertainty due to unknown $\alpha^{3}(Z \alpha)^{4}$ terms. The result of Ref. [21, removes the first uncertainty and our calculation of the three loop slope of the Dirac form factor removes the last one. The total uncertainty in the theoretical predictions in the $1 S$ Lamb shift is therefore reduced to $16 \mathrm{kHz}$.

Turning the problem around, we note that the small theoretical uncertainty on the $1 S$ Lamb shift permits an extraction of the proton charge radius by comparing experimental and theoretical results. We then arrive at the precise value of the proton radius $r_{p}=0.883 \pm 0.014 \mathrm{fm}$.

In conclusion, we have computed the three-loop slope of the Dirac form factor. Thanks to this calculation the theoretical uncertainty in the predictions for the $1 \mathrm{~S} \mathrm{Lamb}$ shift is reduced. Comparison of the theoretical and experimental results for the $1 S$ level shift permits an accurate determination of the proton charge radius. Further improvements in theoretical predictions for the $1 \mathrm{~S}$ level shift would be possible if subleading $\alpha^{2}(Z \alpha)^{6} \log ^{2} \alpha$ corrections are calculated. Only then can the theoretical uncertainty be brought down to several $\mathrm{kHz}$ and can the potential of the recent measurement of the $1 \mathrm{~S}-2 S$ transition frequency [i, be fully exploited.

Acknowledgments: We are grateful to E. Remiddi for useful conversations and to S.G. Karshenboim,
K. Pachucki and A. Yelkhovsky for comments on the manuscript. This research was supported in part by the United States Department of Energy, contract DE-AC03-76SF00515, by BMBF under grant number BMBF-057KA92P, by Graduiertenkolleg "Teilchenphysik" at the University of Karlsruhe and by the DFG Forschergruppe "Quantenfeldtheorie, Computeralgebra und Monte-Carlo-Simulation".

[1] Quantum Electrodynamics, Series (World Scientific, Singapore, 1990), edited by T. Kinoshita.

[2] M. Weitz et al, Phys. Rev. Lett. 72, 328 (1994).

[3] D.J. Berkeland et al., Phys. Rev. Lett. 75, 2470 (1995).

[4] S. Bourzeix et al., Phys. Rev. Lett. 76, 384 (1996).

[5] B. de Beauvoir et al. Phys. Rev. Lett. 78, 440 (1997).

[6] Th. Udem, et al., Phys. Rev. Lett. 79, 2646 (1997).

[7] C. Schwob et al., Phys. Rev. Lett. 82, 4960 (1999).

[8] J.R. Sapirstein and D.R. Yennie, in Ref. ["' 1 , p.560.

[9] P. Mohr, in Atomic, Molecular and Optical Physics Handbook, edited by G.W.F. Drake, pg. 341, (AIP Press, Woodbory, New York, 1996).

[10] K. Pachucki et al., J. Phys.B29, 177 (1996).

[11] S.G. Karshenboim, Soviet Physics JETP 76, 541 (1993).

[12] G. 't Hooft and M. Veltman, Nucl. Phys. B44, 189 (1972); K.G. Chetyrkin and F. Tkachov, Nucl. Phys. B192, 159 (1981).

[13] S. Laporta and E. Remiddi, Phys. Lett. B379, 283 (1996).

[14] T.W. Appelquist and S.J. Brodsky, Phys. Rev. A2, 2293 (1970).

[15] R. Barbieri, J.A. Magnaco and E. Remiddi, Nuovo Cimento Lett. 3, 588 (1970).

[16] We are using the values as advocated by $\mathrm{Na}^{-}$ tional _nsstitute _of _. Standards and Technology. See http: / / www_nist.gov/cuu,

[17] P. Baikov and D.J. Broadhurst, hep-ph/9504398,

[18] K. Pachucki, Phys. Rev.A46 (1992), 648; Ann. Phys. (N.Y.) 226, 1 (1993).

[19] K. Pachucki, Phys. Rev. Lett.72, 3154 (1994).

[20] M. Eides and V. Shelyuto, Phys. Rev.A52, 954 (1995).

[21] U.D. Jentschura, P.J. Mohr and G. Soff, Phys. Rev. Lett. 82, 53 (1999).

[22] E.E. Salpeter, Phys. Rev. 87, 328 (1952).

[23] K. Pachucki and H. Grotch, Phys. Rev. A51, 1854 (1995).

[24] K. Pachucki, Phys. Rev. A52, 1079 (1995).

[25] J.L. Friar et al., Phys. Rev. A59, 4061 (1999).

[26] L.N. Hand et al., Rev. Mod. Phys. 35, 335 (1963).

[27] G.G. Simon et al., 381 (1980).

[28] Ch.W. Wong, Int. J. Mod. Phys. 3, 821 (1994).

[29] S.G. Karshenboim, Can. J. Phys. 77, 241 (1999). 